\title{
Properties of open and hidden charm mesons in light quark matter
}

\author{
Martin Cleven* \\ Departament de Fisica Quantica i Astrofisica and Institut de Ciencies del Cosmos \\ Universitat de Barcelona, 08028-Barcelona, Spain \\ E-mail: clevenefqa.ub.edu

\section{Volodymyr K. Magas} \\ Departament de Fisica Quantica i Astrofisica and Institut de Ciencies del Cosmos \\ Universitat de Barcelona, 08028-Barcelona, Spain \\ E-mail: vladimirefqa.ub.edu

\section{Angels Ramos} \\ Departament de Fisica Quantica i Astrofisica and Institut de Ciencies del Cosmos \\ Universitat de Barcelona, 08028-Barcelona, Spain \\ E-mail: ramosefqa.ub.edu
}

In this work we study the implications of light-quark pionic matter at finite temperatures on the properties of open and hidden charm mesons. The meson-meson interactions are described by means of a chiral unitary approach accounting for coupled channels and the finite temperature effects are included using the Imaginary Time Formalism. The key observables presented here are the spectral function and the pion-induced width at finite temperatures for the mesons $D, D^{*}$ and most importantly $J / \psi$. As a subsequent application the charmed-meson spectral functions are used to describe the behaviour of a dynamically generated $X(3872)$ in a hot pion bath.

XVII International Conference on Hadron Spectroscopy and Structure - Hadron2017

25-29 September, 2017

University of Salamanca, Salamanca, Spain

\footnotetext{
* Speaker.
} 


\section{Introduction}

With a new generation of heavy-ion-collision experiments operational or under construction -LHC, FAIR or NICA- there is a demand for theoretical predictions of hadronic properties at temperatures and densities far from standard nuclear physics scenarios. Among the various subjects, the study of the $J / \psi$ stands out as a candidate to signal deconfinement, according to the Matsui and Satz prediction [1]. A quark-gluon plasma (QGP) produced in the collision would screen the $c \bar{c}$ interaction or ionize the charmonium state. Either way, this would lead to a suppression of events.

However, although in previous experiments such a drop was actually seen [2], it still remains unclear whether this is indeed related to the formation of a QGP.

The inelastic interactions of the $J / \psi$ with the surrounding hadronic medium, accounted for in the 'co-mover' models, offer alternative mechanisms to explain the drop in the $J / \psi$ production that either do not involve the transition to QGP state or, at least, reduce the number of $J / \psi$ during the evolution in the hadronic phase of reaction, and thus have to be taken into account.

Obtaining the properties of the $D$ and $D^{*}$ mesons in hot pionic matter, which we consider to be the first level approximation of the matter generated in ultrarelativistic heavy ion collision, will be one of the main topics of the present study. While interesting in itself, the study of these mesons is also closely related to that of the $J / \psi$ through coupled-channel effects. Therefore, the second focus will be on studying the properties of the hidden charm $J / \psi$ meson under the same conditions. Such a combined effort will provide a comprehensive understanding of how the properties of open and hidden charm mesons are modified when interacting with pionic matter at non-vanishing temperatures.

With the spectral functions for charmed mesons we are also able to study the behaviour of the $X(3872)$ under the assumption that it is formed from charmed meson interactions. This provides an additional tool in determining its true nature.

\section{Theoretical Framework}

We start by briefly recalling the main features of meson-meson scattering in a chiral $S U(4)$ as used in Ref. [3]. For further reading see e.g. Refs. [4, 5]. Here the potentials for pseudoscalarpseudoscalar and pseudoscalar-vector scattering will be derived from the Lagrangians

$$
\mathscr{L}_{\mathrm{PPPP}}=\frac{1}{12 f^{2}}\left\langle J^{\mu} J_{\mu}+\Phi^{4} M\right\rangle \quad \mathscr{L}_{\mathrm{VPVP}}=-\frac{1}{4 f^{2}}\left\langle J^{\mu} \mathscr{J}_{\mu}\right\rangle,
$$

where the vector currents for pseudoscalar and vector mesons are given by

$$
J_{\mu}=\left(\partial_{\mu} \Phi\right) \Phi-\Phi\left(\partial_{\mu} \Phi\right), \quad \mathscr{J}_{\mu}=\left(\partial_{\mu} \mathscr{V}_{v}\right) \mathscr{V}^{v}-\mathscr{V}_{v}\left(\partial_{\mu} \mathscr{V}^{v}\right)
$$

respectively, with the $S U(4)$ pseudoscalar and vector multiplets $\Phi$ and $\mathscr{V}_{\mu}$, respectively. This allows us to calculate the Weinberg-Tomozawa-like potentials for the coupled channel interactions $D \pi, D \eta$ and $D^{*} \pi, D^{*} \eta$ in the open-charm sector and $J / \psi \pi, \eta_{c} \rho, D \bar{D}^{*}+c . c$. in the hidden-charm one. For a detailed discussion of the amplitudes we refer to Ref. [3]. At this point we only state the most striking feature in the hidden-charm sector. All interactions that do not contain the charmed meson pair vanish at the order we are studying including $J / \psi \pi \rightarrow J / \psi \pi$. This has important implications 
as will be discussed further down the line. The $S$-wave projections of these amplitudes $V$ can be used as the kernel for the Lippmann-Schwinger Equation. Using the on-shell formalism this simplifies to a simple algebraic equation that can easily be solved as $T=(1-V G)^{-1} V \vec{\varepsilon} \cdot \vec{\varepsilon}^{\prime}$

$$
T=(1-V G)^{-1} V \vec{\varepsilon} \cdot \vec{\varepsilon}^{\prime},
$$

for the scattering of vector mesons off pseudoscalar ones, while for the purely pseudoscalar case one simply needs to remove the polarization vectors $\vec{\varepsilon}, \vec{\varepsilon}^{\prime}$ from the previous equation. The diagonal matrix $G$ contains the two-meson loops calculated using dimensional regularization. For more details on this see Refs. [4, 5].

From here on we focus on how the meson-meson interactions are modified by a surrounding pion medium at finite temperatures. For brevity we will focus on the main results and refer to Ref. [3] and references therein for more details. The modifications will be two-fold. First, we will use the Imaginary Time Formalism (ITF) to incorporate the impact of finite temperatures. This will be done by modifying the propagators and through the loops also the unitarized amplitudes. Secondly, we will dress the propagators for the mesons $M$ with the self energy obtained from closing the pion line in the $M \pi \rightarrow M \pi$ scattering amplitude. This way the dressed propagators account for the pion bath. As these dressed propagators affect the amplitudes, the process needs to be iterated several times to be self-consistent.

In ITF the zeroth components of the four-momenta are modified using $q_{0} \rightarrow \mathrm{i} \omega_{n}=\mathrm{i} 2 \pi n T$ with the discrete Matsubara frequencies $\omega_{n}=\mathrm{i} 2 \pi n T$ and replacing the zeroth component of the loop integrals with a sum over these frequencies. As mentioned above, in ITF the interactions remain unchanged and only the propagators are modified. The meson propagator becomes

$$
D_{M}\left(\omega_{n}, \vec{q} ; T\right)=\left[\left(\mathrm{i} \omega_{n}\right)^{2}-\vec{q}^{2}-m_{M}^{2}-\Pi_{M}\left(\omega_{n}, \vec{q} ; T\right)\right]^{-1}
$$

Once the sums over the Matsubara frequencies are performed one may analytically continue the result to external frequencies $\omega+\mathrm{i} \varepsilon$. In the case of the two-meson loop at finite temperature we find

$$
G_{M M^{\prime}}\left(p^{0}, \vec{p} ; T\right)=\int \frac{\mathrm{d}^{3} q}{(2 \pi)^{3}} \int \mathrm{d} \omega \int \mathrm{d} \Omega \frac{S_{M}(\omega, \vec{q} ; T) S_{M^{\prime}}(\Omega, \vec{p}-\vec{q} ; T)}{p^{0}-\omega-\Omega+\mathrm{i} \varepsilon}[1+f(\omega, T)+f(\Omega, T)]
$$

Here $f(\omega, T)=[\exp (\omega / T)-1]^{-1}$ is the meson Bose distribution function at temperature $T$ and $S_{M}\left(\omega_{n}, \vec{q} ; T\right)=-(1 / \pi) \operatorname{Im}\left(D_{M}\left(\omega_{n}, \vec{q} ; T\right)\right)$ is the spectral function of the meson $M$. For details of the regularization and a more detailed discussion of how the finite temperatures affect the individual loops and, consequently through unitarization, the amplitudes see Ref. [3].

The self energy of a meson $M$ is obtained from closing the pion line in the $M \pi \rightarrow M \pi T$-matrix element $T_{M \pi}$. After applying the ITF Feynman rules and carrying out the Matsubara sum we find for real energies $p^{0}$

$$
\Pi_{M}\left(p^{0}, \vec{p}\right)=\int \frac{\mathrm{d}^{3} q}{(2 \pi)^{3}} \int \mathrm{d} \Omega \frac{f(\Omega, T)-f\left(\omega_{\pi}, T\right)}{\left(p^{0}\right)^{2}-\left(\omega_{\pi}-\Omega\right)^{2}+\mathrm{i} \varepsilon}\left(-\frac{1}{\pi}\right) \operatorname{Im} T_{M \pi}(\Omega, \vec{p}+\vec{q} ; T) .
$$



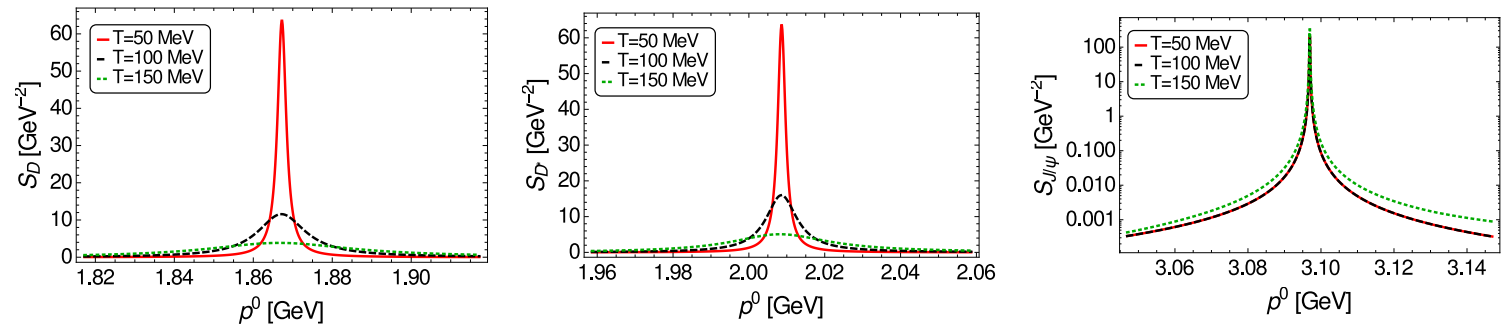

Figure 1: Spectral functions of $D, D^{*}$ and $J / \psi$ at temperatures $T=50,100,150 \mathrm{MeV}$. Notice that for $D$ and $D^{*}$ we use a linear, for $J / \psi$ a logarithmic plot.

\section{Results}

In Fig. 1 we show the spectral functions of $D, D^{*}$ and $J / \psi$ resulting from their interaction with the hot pion bath as a function of the energy at increasing temperatures $T=50,100,150 \mathrm{MeV}$. In the case of the charmed mesons we see how the strength spreads considerably when increasing the temperature from 50 to $150 \mathrm{MeV}$. In other words, the spectral function of the charmed mesons broadens significantly in a finite-temperature pion bath. The situation is obviously different for the $J / \psi$ spectral function. Notice that in this case we have also included the $J / \psi$ vacuum width of $\Gamma_{J / \psi}^{\mathrm{Vac}}=(93 \pm 3) \mathrm{keV}[6]$. On one hand this makes the numerical determination of the spectral function easier, on the other hand it gives an intuitive measure of the impact of the pion-induced width. The large self-energy for the charmed mesons made this incorporation irrelevant there.

The $J / \psi$ spectral functions at the temperatures $T=50 \mathrm{MeV}$ and $T=100 \mathrm{MeV}$ are indistinguishable from the vacuum one. One needs to go to temperatures as large as $T=150 \mathrm{MeV}$ to start seeing noticeable broadening of the $J / \psi$ spectral function compared to its free case shape. The reason for this is basically two-fold. First, all interactions that contribute to the $J / \psi$ are weak which leads to a lower self energy. Secondly, due to the absent leading interaction for $J / \psi \pi$ scattering this process has to go through a charmed meson pair. This, however, corresponds to a significantly larger threshold and has therefore less impact at energies close to the mass of the $J / \psi$.

While a broadening spectral function for $T=150 \mathrm{MeV}$ can be seen from our results, it still remains similar to the vacuum case. From this we can conclude that the $J / \psi$ retains most of its shape in hot pionic matter. A suppression in the detection of $J / \psi$ mesons in a hot pionic medium should therefore not be attributed to the interaction with the surrounding hadrons. Thus, if observed, such a drop might indeed point towards the detection of a QGP.

To demonstrate the impact of increasing temperatures we use a more illustrative quantity, the width of the mesons $M$ obtained from the surrounding pion

$$
\Gamma_{M}=\operatorname{Im} \Pi_{M}\left(p^{0}=m_{M}, \vec{p}=0\right) / m_{M} .
$$

Fig. 2 shows the temperature behaviour of $\Gamma_{M}$ for the three mesons in question, $D, D^{*}$ and $J / \psi$. Let us discuss the final results which correspond to the last iteration at each sub-figure of Fig. 2, namely $n=3$ for the charmed meson case and $n=4$ for $J / \psi$ (see Ref. [3] for more details on iterations). In the case of the charmed mesons we see a slow rise in the single $\mathrm{MeV}$ region for temperatures below $100 \mathrm{MeV}$ and a sharper rise beyond that up to around $70-90 \mathrm{MeV}$ for temperatures approaching 
(a)

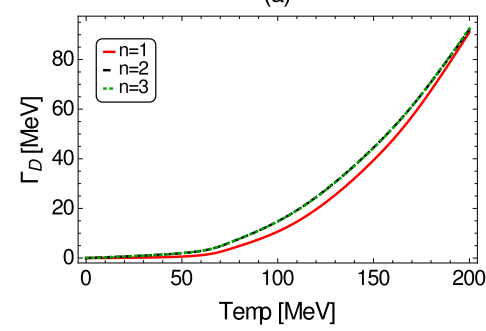

(b)

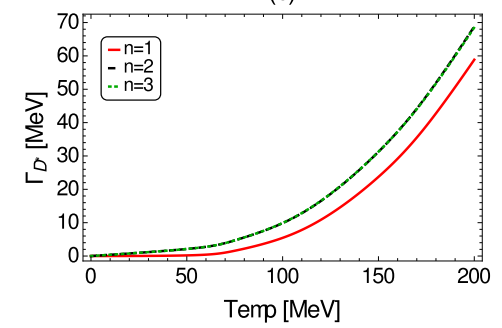

(c)

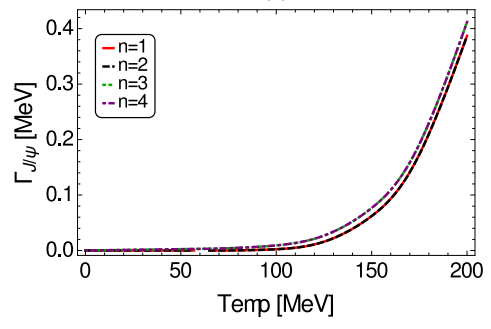

Figure 2: We show the pion-induced width as a function of the temperature. To illustrate the process of achieving self consistent results we show this for different iterations $n$.

(a)

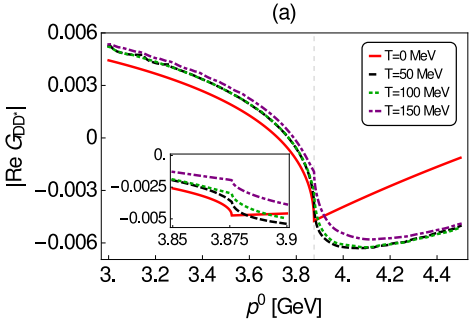

(b)

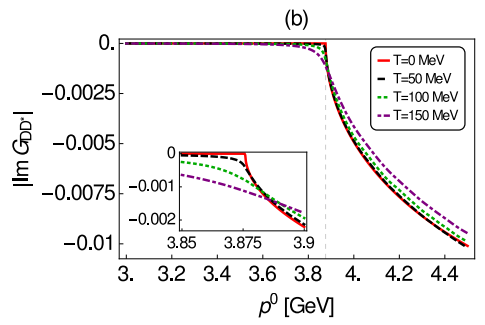

(c)

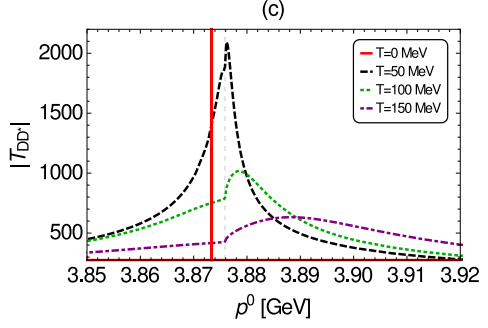

Figure 3: Real part of the $D D^{*}$ loop (left panel), imaginary part of the $D D^{*}$ loop (central panel), absolute value of the unitarized amplitude for $D D^{*}$ scattering. All quantities are shown at temperatures $0,50,100,150 \mathrm{MeV}$. In the right panel we use a vertical line to represent the $X(3872)$ at $T=0 \mathrm{MeV}$.

$200 \mathrm{MeV}$. Compared to the hadronic width of the $D^{*}$ in the vacuum of the order of $100 \mathrm{keV}$ this means an increase of 2-3 orders of magnitude depending on the temperature. The general behaviour is similar for the $J / \psi$ albeit suppressed by 2-3 orders of magnitude. Only at temperatures around $150 \mathrm{MeV}$ the pion induced width exceeds the vacuum width $\Gamma_{J / \psi}^{\mathrm{Vac}}=(93 \pm 3) \mathrm{keV}$ [6].

As a further application of the spectral functions we study the $X(3872)$. In our model the $X(3872)$ is a dynamically generated state in the channels $D^{*} \bar{D}+$ c.c. and $D_{s}^{*} \bar{D}_{s}+$ c.c. with $I^{G}\left(J^{P C}\right)=0^{+}\left(1^{++}\right)$and is dominated by the former. So, naturally, it has to be sensitive to they way the charmed mesons are modified in a hot pionic medium.

The left and central panel of Fig. 3 show the real and imaginary part of the $D D^{*}$ loop, respectively. The changes to the loop are not dramatic, yet, interestingly, the most pronounced changes at least for the imaginary part - occur in the region right below threshold (c.f. the zoom-in shown in the inlet). This has implications for an $X(3872)$ that is generated in unitarized amplitudes with these loops. The non-vanishing imaginary part below threshold means that the state obtains additional width, the change in the real part moves the peak position. Our results for the unitarized amplitude for $D D^{*}$ scattering at various temperatures are shown in the right panel of Fig. 3.

The behaviour of the $X(3872)$ presented here is a unique feature of the molecular interpretation and is due to the relatively strong interactions of the charmed mesons with the pion bath. A conventional charmonium $\chi_{c 1}(2 P)$ or a compact tetra quark state would barely change its behaviour under the same circumstances. So an experimental confirmation of this could be seen as a strong hint that the $X(3872)$ has a dominant molecular component. 


\section{Summary}

We have calculated the behaviour of the charmed mesons and the $J / \psi$ in a pion bath at finite temperatures using unitarized $S U(4)$ chiral amplitudes. Using the ITF formalism we obtained observables that are relevant for understanding the behaviour of mesons containing charm quarks in hot mesonic matter, most notably the induced width and spectral function.

We find that the charmed $D$ and $D^{*}$ mesons acquire a substantial width, reaching values in the $30-40 \mathrm{MeV}$ range at $T=150$.

The modification of the $J / \psi$ meson properties by a hot pion bath is substantially more moderate, even at temperatures of around $T=150 \mathrm{MeV}$ the $J / \psi$ retains most of its shape in hot pionic matter. Consequently, an observed drop in $J / \psi$ detection should indeed point towards the formation of a QGP, the interaction with the surrounding hadrons being essentially negligible.

Our results can be used as input in theoretical simulations of open and hidden charm meson propagation in a hot pion gas, aiming at understanding the transition from a hadronic to a QGP phase.

As an application of the spectral functions we have calculated the behaviour of an $X(3872)$ generated from charmed meson interactions and shown that in this picture the state becomes broader while its position slightly moves.

Acknowledgements: This work is partly supported by the Spanish Ministerio de Economia y Competitividad (MINECO) under the project MDM-2014-0369 of ICCUB (Unidad de Excelencia 'María de Maeztu'), and, with additional European FEDER funds, under the contract FIS2014-54762-P and the Spanish Excellence Network on Hadronic Physics FIS2014-57026-REDT and from the Generalitat de Catalunya contract 2014SGR-401.

\section{References}

[1] T. Matsui, H. Satz, Phys. Lett. B 178 (1986) 416.

[2] M. C. Abreu et al. [NA50 Collaboration], Phys. Lett. B 450, 456 (1999); Phys. Lett. B 477, 28 (2000).

[3] M. Cleven, V. K. Magas and A. Ramos, Phys. Rev. C 96 (2017) no.4, 045201 doi:10.1103/PhysRevC.96.045201 [arXiv:1707.05728 [hep-ph]].

[4] D. Gamermann, E. Oset, D. Strottman and M. J. Vicente Vacas, Phys. Rev. D 76 (2007) 074016 doi:10.1103/PhysRevD.76.074016 [hep-ph/0612179].

[5] D. Gamermann and E. Oset, Eur. Phys. J. A 33 (2007) 119 doi:10.1140/epja/i2007-10435-1 [arXiv:0704.2314 [hep-ph]].

[6] C. Patrignani et al. [Particle Data Group], Chin. Phys. C 40, no. 10, 100001 (2016). 